\title{
Unveiling the circumstellar environment toward a massive young stellar object ${ }^{\star}$
}

\author{
S. Paron ${ }^{1,2}$, C. Fariña ${ }^{3}$, and M. E. Ortega ${ }^{1}$ \\ 1 Instituto de Astronomía y Física del Espacio (IAFE), CC 67, Suc. 28, 1428 Buenos Aires, Argentina \\ e-mail: [sparon;mortega]@iafe.uba.ar \\ 2 FADU and CBC, Universidad de Buenos Aires, 1428 Buenos Aires, Argentina \\ 3 Isaac Newton Group of Telescopes, 38700 La Palma, Spain \\ e-mail: cf@ing.iac.es
}

Received 21 August 2013 / Accepted 7 October 2013

ABSTRACT

\begin{abstract}
Aims. As a continuation of a previous work, in which we found strong evidence of massive molecular outflows toward a massive starforming site, we present a new study of this region based on very high angular resolution observations with the aim of discovering the outflow-driven mechanism.

Methods. Using near-IR data acquired with Gemini-NIRI at the broad $H$ - and $K$ s-bands, we studied a region of $22^{\prime \prime} \times 22^{\prime \prime}$ around the UCHII region G045.47+0.05, a massive-star forming site at a distance of about $8 \mathrm{kpc}$. To image the source with the highest spatial resolution possible we employed the adaptative optics system ALTAIR, achieving an angular resolution of about 0 .' 15 .

Results. We discovered a cone shaped nebula that has an opening angle of about $90^{\circ}$ and extends eastward of the IR source $2 \mathrm{MASS}$ $\mathrm{J} 19142564+1109283$, which is very likely a massive young stellar object (MYSO). This morphology suggests a cavity that was cleared in the circumstellar material, and its emission may arise from scattered continuum light, warm dust, and probably also from emission lines from shock-excited gas. The nebula, which presents arc-like features, is connected with the IR source through a jet-like structure, which is aligned with the blueshifted CO outflow found in a previous study. The near-IR structure lies $\sim 3^{\prime \prime}$ north of the radio continuum emission, revealing that it is not spatially coincident with the UCHII region. The observed morphology and structure of the near-IR nebula strongly suggest the presence of a precessing jet. We resolved the circumstellar environment (in scale of a thousand $\mathrm{AU}$ ) of a distant MYSO, indeed one of the farthest currently known.
\end{abstract}

Key words. stars: formation - ISM: jets and outflows - HII regions

\section{Introduction}

The formation of high-mass stars plays a very important role in the evolution and dynamics of the Galaxy. Despite the high impact that they have on the environment, the physical processes involved in their formation are less well understood than those of their low-mass counterpart. The birth of a massive star is a violent event that produces intense extreme ultraviolet radiation that ionizes the surroundings (e.g. Hester \& Desch 2005; Peters et al. 2010). The formation of a massive star also produces jets and massive molecular outflows (e.g. Wu et al. 2004; Vaidya et al. 2011), which may contribute to the removal of excess angular momentum from accreted matter and to disperse infalling circumstellar envelopes (Reipurth \& Bally 2001; Preibisch et al. 2003). Such a phenomenon can take place even when the object has reached the ultracompact HII (UCHII) region stage (Hunter et al. 1997; Qin et al. 2008). Taking into account that high-mass stars generally do not form in isolation but in dense clusters and that the sites of massive star formation are located at far distances, very high angular resolution imaging is required to reveal the morphologies of their circumstellar ambient.

* Reduced NIR data (FITS) are only available at the CDS via anonymous ftp to cdsarc.u-strasbg. fr (130.79.128.5) or via http://cdsarc.u-strasbg.fr/viz-bin/qcat?]/A+A/559/L2
The UCHII region G045.47+0.05 (hereafter G45.47) is located toward the eastern border of the extended HII region G45L (Paron et al. 2009) and is deeply embedded in a dense and dark molecular cloud. Figure 1 displays a three-color SpitzerIRAC image of the large field where G45.47 is located; the white box indicates its position. The IRAC data were extracted from the GLIMPSE Survey (Benjamin et al. 2003). G45.47 is included in the extensive study of UCHII regions of Wood \& Churchwell (1989), who adopted a distance of $9.7 \mathrm{kpc}$ for it. According to Paron et al. (2009), the HII region G45L is located at a distance of about $8 \mathrm{kpc}$, same as the UCHII region complex G45.45+0.06 (Kuchar \& Bania 1994), which is the brightest structure in Fig. 1. By considering that all these sources belong to the same complex, Ortega et al. (2012) adopted a distance of $8.3 \mathrm{kpc}$ for G45.47. However, following Araya et al. (2002), distances as close as $6 \mathrm{kpc}$ cannot be excluded for this source. Class II $\mathrm{CH}_{3} \mathrm{OH}$ maser emission at $6.6 \mathrm{GHz}$ was detected toward G45.47 (Caswell et al. 1995) with a central velocity of $56 \mathrm{~km} \mathrm{~s}^{-1}$, which agrees with the systemic velocities of the HII region complex and the related molecular cloud. It is known that this maser is radiatively pumped by IR emission from the warm dust associated with massive young stellar objects (MYSOs). Indeed, in a previous study, Cesaroni et al. (1992), using $\mathrm{NH}_{3}$ line observations, found evidence of collapse toward G45.47. Based on high-angular resolution $\left(\sim 5^{\prime \prime}\right)$ molecular line observations, Wilner et al. (1996) identified several $\mathrm{HCO}^{+} J=1-0$ clumps 


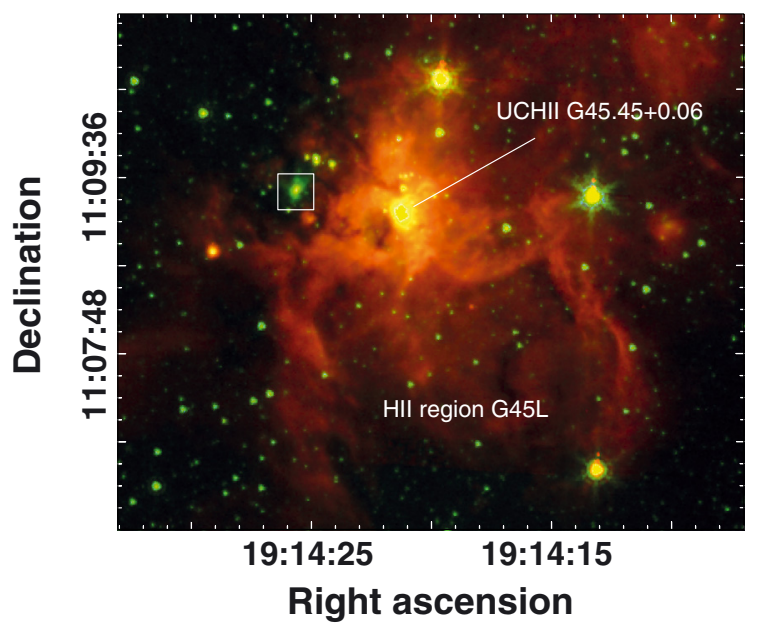

Fig. 1. Three-color Spitzer-IRAC image of a large field where the UCHII region G45.47 is located ( $8 \mu \mathrm{m}$ in red, $4.5 \mu \mathrm{m}$ in green, and $3.5 \mu \mathrm{m}$ in blue). The white box indicates the field observed with Gemini-NIRI and G45.47 is the extended green source within it.

which suggests that G45.47 is in the early stages of forming an OB cluster. G45.47 is associated with an extended source seen at $4.5 \mu \mathrm{m}$ in Spitzer-IRAC images, the "extended green object" EGO G45.47+0.05, that was cataloged by Cyganowski et al. (2008) as a "likely" MYSO outflow candidate. Recently, Ortega et al. (2012), using intermediate angular resolution observations from several molecular transitions, characterized the dense molecular clump where G45.47 is embedded and found strong evidence of outflow activity in the region.

In this study, we report the results obtained from near-IR observations performed with Gemini-NIRI with high-angular resolution toward $\mathrm{G} 45.47$.

\section{Observations and data reduction}

In this study we analyzed the $H$ and $K$ s broad-band images of the UCHII region G45.47 acquired with NIRI (Hodapp et al. 2003) at Gemini-North. The observations were carried out during April and June 2012 in queue mode (program GN-2012A-Q-20). NIRI was used with the $f / 32$ camera which provides a plate scale of $0{ }^{\prime} 022 \mathrm{pix}^{-1}$ in a field of view of $22^{\prime \prime} \times 22^{\prime \prime}$. The white box in Fig. 1 represents the field observed with NIRI centered at $\mathrm{RA}=19^{\mathrm{h}} 14^{\mathrm{m}} 25.6^{\mathrm{s}}$, Dec $=+11^{\circ} 09^{\prime} 28.5^{\prime \prime}, \mathrm{J} 2000$. To image the source with the highest spatial resolution possible, NIRI was used together with the Gemini facility adaptative optics (AO) system, ALTAIR (Herriot et al. 2000; Boccas et al. 2006). We needed to use the laser guide star system (LGS) for the AO (with a natural guide star only for tip/tilt correction) because G45.47 is deeply embedded in a dense molecular clump with high extinction and it was not possible to find a nearby star that fulfilled the $\mathrm{AO}$ brightness requirement in the optical $V$-band. The available natural tip/tilt star was offset from the UCHII region as well and was relatively faint, which resulted in a slight elongation of the PSF, noticeable mainly in the $H$-band. The images in $H$ - and $K$ s-band were performed using a dither pattern with offsets to sky fields. The images were reduced using standard procedures for near-IR imaging provided by the Gemini Observatory through the IRAF-NIRI package. The effective seeing was $\sim 0{ }^{\prime} 15$ (measured as the FWHM of point sources in the field of the final co-added images). After excluding several individual frames with anomalous electronic noise and with evident poor-quality PSFs, the effective exposure times of the final

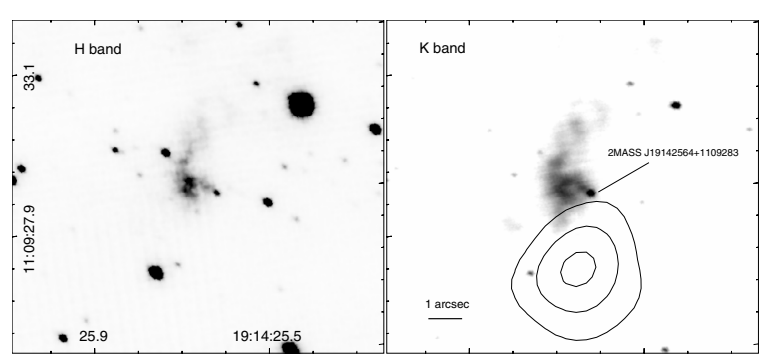

Fig. 2. Left: $H$-band emission toward G45.47. Right: same field displaying the Ks-band emission. The point source 2MASS $19142564+1109283$ is indicated and contours of the radio continuum emission at $6 \mathrm{~cm}$ are included with levels of 5,20 , and $50 \mathrm{mJy} \mathrm{beam}^{-1}$.

co-added images were $50 \mathrm{~s}$ and $120 \mathrm{~s}$ for the $K \mathrm{~s}$ - and $H$-band, respectively. The signal-to-noise ratio of the extended emission is $\sim 20$ for the $K$ s-band and $\sim 7$ for the $H$-band. The absolute astrometry in NIRI images was performed using three near-IR stars in the field from the 2MASS Point Source Catalog; fortunately, the three stars were well distributed in the field, which yielded a good astrometrical solution.

\section{Results and discussion}

The broad $H$-band emission obtained with Gemini-NIRI toward G45.47 is presented in the left panel of Fig. 2, while the right panel displays the broad $K$ s-band emission of the same field. Both images were slightly smoothed to better display the diffuse emission that appears at the center of each panel and eastward of the point source 2MASS J19142564+1109283. This IR source was suggested to be an early B-type YSO by Ortega et al. (2012). The diffuse emission, better appreciated at the $K$ s band, shows a nebula with a cone shape displaced $\sim 3^{\prime \prime}$ northward of the peak of the radio continuum emission at $6 \mathrm{~cm}$, which is displayed with contours. The radio continuum data were extracted from the RMS Survey (Urquhart et al. 2009) and have a synthetized beam size of about 1'.7 This displacement was previously found by De Buizer et al. (2005), who used mid-IR data with a resolution $>1$ '. 2 , they could not fully discard an astrometric problem. Our observations confirm that the near-IR structure does not coincide with the radio continuum emission, which testifies to the complexity of this massive star-forming region. Thus, as a first important result, we conclude that the UCHII region G45.47 is not directly related to 2MASS J19142564+1109283 and its associated near-IR nebula. This is a similar case as found toward the hot molecular core G9.62+0.19, where some UCHII regions, bright in radio continuum, have no counterpart in the near-IR (Linz et al. 2005). It seems to be common to find secondary cores slightly separated from UCHII regions (e.g. Beuther et al. 2007).

Figure 3 shows a zoom-in of the Ks-band image with some emission contours to better display the morphology. The cone shaped nebula, with an opening angle of about $90^{\circ}$, presents arclike features with concave faces pointing toward the IR source. These features seem to be connected to the IR source by a jetlike structure that is aligned with the blueshifted $\mathrm{CO}$ outflow found by Ortega et al. (2012). Figure 4 presents the Ks-band emission with contours displaying the blueshifted ${ }^{12} \mathrm{CO} J=3-2$ outflow and the $4.5 \mu \mathrm{m}$ IRAC emission. In the image, the blue arrow indicates the mentioned alignment. Therefore, we suggest that the $\mathrm{CO}$ outflows are not related to the UCHII region G45.47. The driving source of the outflows is probably 2MASS J19142564+11092832. 


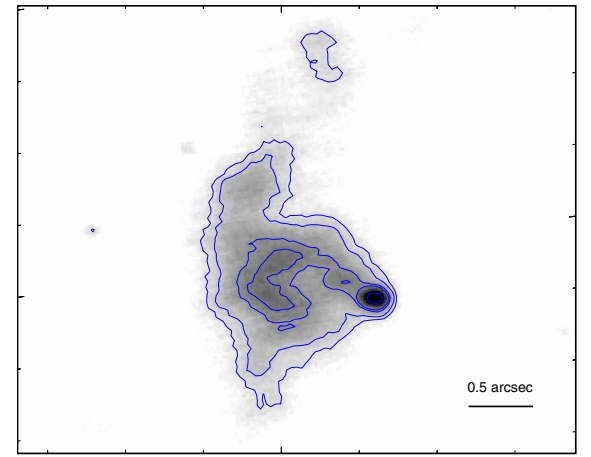

Fig. 3. Zoom-in of the Ks-band emission showing the cone shaped nebula. Contours were included to better appreciate the morphology.

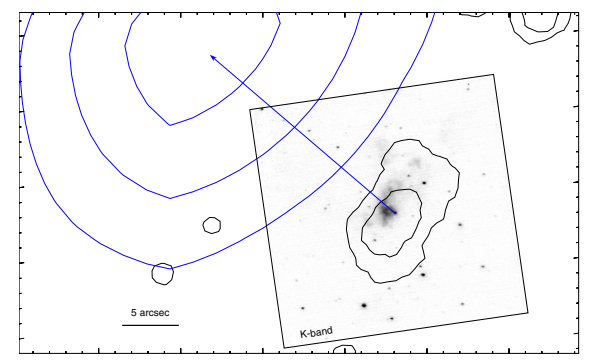

Fig. 4. The box shows the field observed with Gemini-NIRI and displays the broad $K$ s-band emission. The blue contours are the blueshifted CO outflow found by Ortega et al. (2012) and the black ones represent the $4.5 \mu \mathrm{m}$ IRAC emission that delimits the EGO G45.47+0.05 boundaries. The angular resolution of the $\mathrm{CO}$ emission is about $20^{\prime \prime}$. The blue arrow shows the alignment between the structure that connects the IR source with the near-IR arc-like features and the blueshifted CO outflow.

The wide angle $\left(\sim 90^{\circ}\right)$ of the cone shaped nebulosity that points to the blueshifted molecular outflow strongly suggests that the IR emission arises from a cavity cleared in the circumstellar material. The diffuse nebulosity seen in the Ks band may be due to a combination of different emitting processes: continuum emission from the central protostar that is scattered at the inner walls of a cavity, emission from warm dust, and probably line emission from shock-excited $\mathrm{H}_{2}$, among other emission lines (e.g. Bik et al. 2006). The $H$-band emission shown in Fig. 2 (left), which presents a similar morphology as the Ks-band image, may also arise from scattered light and warm dust, and additionally from excited [FeII], tracer of the innermost part of jets that are accelerated near the driving source (Reipurth et al. 2000). It is important to note that we did not detect a similar feature related to the redshifted molecular outflow detected by Ortega et al. (2012). This may be because the redshifted component is probably much fainter than the blueshifted one because it is pointing away from us, which implies a higher extinction. Several studies show similar nebulosities related to MYSOs that are associated only with the blueshifted outflow cavity (e.g. Preibisch et al. 2003; Kraus et al. 2006; Weigelt et al. 2006).

An outstanding unsolved problem concerning jet-driven outflow models is how highly collimated jets can produce the wideangle outflow cavities that are usually observed in massive protostellar envelopes (Mundt et al. 1990; Konigl \& Pudritz 2000). Two possible scenarios have been proposed to explain this. One of them proposes a wide-angle structure produced by the winds of the YSO with a density enhancement along the axis that exhibits a collimated jet-like appearance (Shu et al. 1995; Reipurth \& Bally 2001). Weigelt et al. (2006) found that the

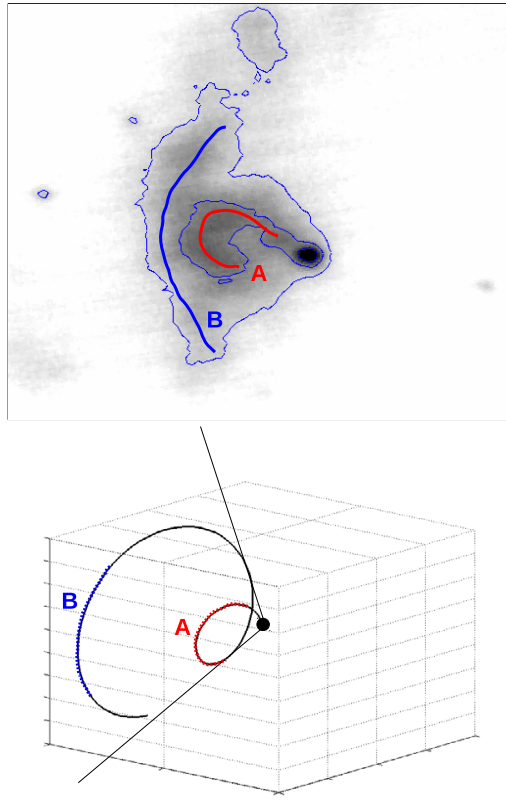

Fig. 5. Upper panel: Ks-band emission toward G45.47. Some contours are superimposed and two arc-like features are marked in red (feature A) and blue (feature B). Bottom panel: sketch of a probable scenario: a precessing jet moving toward us describes an anticlockwise helicoid. The observed arc-like features A and B are marked.

cavity opening angle increases with increasing luminosity of the central source, supporting the hypothesis that a wide-angle stellar wind plays an important role in driving the outflows. In the second scenario, the wide-angle cavities are proposed to be excavated by the action of collimated precessing jets. Kraus et al. (2006) suggested that a precessing jet might explain the difference between the outflow widths observed toward low- and highmass YSOs, adding support to a common collimation mechanism. According to the authors, the precession of a jet may be produced in a binary system where the rotational axis of the jetdriving star is misaligned with the orbital plane of its companion, or may be due to anisotropic accretion events that alter the angular-momentum vector of the protostar disk.

The wide-angle stellar wind and precessing jet models predict different geometries, which can be observationally distinguished. In the former model, the collimated jet-like structure is seen along the flow axis and the emission of the nebulosity structures exhibits a highly axial symmetry. On the other hand, in the precessing jet model it is expected to observe asymmetries between the jet position and the emission from the nebulosity because the jet is free to move through the cavity. In our observations, the most prominent arc-like structure (named A in Fig. 5, upper panel) is connected to the IR source through an elongated feature that probably traces the most recently ejected material from the protostar. A clear asymmetry between the axis of the arc-like feature and the elongated feature is observed, which makes a wide-angle stellar wind scenario unlikely. A less conspicuous arc-like structure (the base of the cone-like structure; named B in Fig. 5, upper panel) can be observed toward the east. This portion of the nebulosity is clumpy and its concavity points to the IR source as in the case of feature A. Thus, the morphology of the observed nebula suggests a spiraling shape produced by a precessing jet. Moreover, the isolated fragment detected toward the north could be associated with the arc-like feature $\mathrm{B}$, showing the asymmetric nature of the cavity and 
supporting the interpretation of a precessing jet. In Fig. 5, bottom panel, we show the arc-like features named A and B in an sketch of an anti-clockwise helicoid, with an angle of $50^{\circ}$ with respect to the plane of the sky. Following the models presented in Smith \& Rosen (2005), who stated that the dominant physical structure for all precessing jets is an inward-facing cone, we suggest that the arc-like morphology observed in our $K \mathrm{~s}$ band image is consistent with an slow precessing jet. Smith \& Rosen (2005) showed that a fast-precessing jet (precession period $=50 \mathrm{yr}$ ) is rapidly disrupted into many bow shocks, while a slow-precessing jet (precession period $=400 \mathrm{yr}$ ) leads to helical flows, generating an spiral shaped nebula. Following the authors, the observed fragmented helicoid structure in our $K$ s-band image can obey the pulsating intrinsic nature of the precessing jet or inhomogeneities of the circumstellar material.

Binary systems can be found in different stellar evolution stages. For example, Connelley et al. (2008) found that for Class I YSOs, the binary frequency of systems with separations between 100 and $4500 \mathrm{AU}$ is about 43\%. Chini et al. (2012) showed high binarity statistics for O- and B-type stars. As previously mentioned, in binary protostar systems with a misaligned disk, a jet precession is expected because of the tidal interactions between companions (e.g. Papaloizou \& Terquem 1995). Using the relation given in Bate et al. (2000), $P_{\text {prec }} \sim 20 \times P_{\text {bin }}$, where $P_{\text {prec }}$ and $P_{\text {bin }}$ are the precession and the binary orbital periods, respectively, and assuming a slow precessing period of $400 \mathrm{yr}$, a $P_{\text {bin }} \sim 20 \mathrm{yr}$ is derived. From the generalization of Kepler's third law (see Eq. (12.15) from Stahler \& Palla 2005) and considering a relatively large binary-system mass of $50 M_{\odot}$, we derive a maximum separation of 27 AU between the system components. With the angular resolution in our image of $0 . ' 15$ (1200 AU at a distance of $8 \mathrm{kpc}$ ) we were unable to spatially resolve a possible binarity under these assumptions. Furthermore, we cannot discard the possibility that there are deeply embedded sources not detected at near-IR, whose existence could be revealed at longer wavelengths (see Linz et al. 2005). Therefore we conclude that binary-induced jet precession may be a plausible scenario. However, we cannot rule out that anisotropic accretion events are responsible for the jet precession. Spectroscopic observations are required to discern between the two scenarios.

\section{Summary}

Using the NIRI instrument at the Gemini North Telescope and the adaptative optics system ALTAIR, we studied the near-IR emission from the UCHII region G045.47+0.05, a massive starforming site located at a distance of about $8 \mathrm{kpc}$. Achieving an angular resolution of 0 . $^{\prime} 15$, we obtained $H$ - and $K$ s-band images with a high level of detail, which allowed us to unveil the driving mechanism of the massive molecular outflows found in a previous study.

We found near-IR diffuse emission with a cone shape extending eastward of the IR source 2MASS J19142564+1109283, which is very likely a MYSO. This morphology suggests a cavity that was cleared in the circumstellar material, and its emission may arise from a combination of different emitting processes: continuum emission from the central protostar that is scattered at the inner walls of a cavity, emission from warm dust, and probably emission lines from shock-excited gas. The cone-shaped nebula, with an opening angle of about $90^{\circ}$, presents some arclike features whose concave faces point toward the IR source. These features seem to be connected to the IR source by a jetlike structure aligned with the blueshifted $\mathrm{CO}$ outflow found in a previous study. The near-IR structure lies $\sim 3^{\prime \prime}$ north of the radio continuum emission, revealing that it is not spatially coincident with the UCHII region and strongly suggesting that the outflows are not generated by it. The driving source of the outflows is very probably 2MASS J19142564+11092832. Taking into account the observed asymmetry between the axis of the arc-like features and the elongated structure that connects them to the IR source, we proposed that the spiraling shape of the nebula is produced by a precessing jet. The observed fragmented helicoid structure may be caused by the pulsating intrinsic nature of the precessing jet or inhomogeneities of the circumstellar material. We proposed that the jet precession may be due to tidal interactions in an unresolved binary system or to anisotropic accretion events occurring in one MYSO. Spectroscopic observations are required to discern between the two scenarios. We conclude that we resolved the circumstellar environment of a distant (6-9 kpc) MYSO, indeed one of the farthest cases.

Acknowledgements. We would like to thank the anonymous referee for her/his extremely helpful comments. S.P. and M.O. are members of the Carrera del investigador cientifico of CONICET, Argentina. This work was partially supported by grants awarded by CONICET, ANPCYT and UBA (UBACyT).

\section{References}

Araya, E., Hofner, P., Churchwell, E., \& Kurtz, S. 2002, ApJS, 138, 63 Bate, M. R., Bonnell, I. A., Clarke, C. J., et al. 2000, MNRAS, 317, 773 Benjamin, R. A., Churchwell, E., Babler, B. L., et al. 2003, PASP, 115, 953

Beuther, H., Zhang, Q., Bergin, E. A., et al. 2007, A\&A, 468, 1045

Bik, A., Kaper, L., \& Waters, L. B. F. M. 2006, A\&A, 455, 561

Boccas, M., Rigaut, F., Bec, M., et al. 2006, in SPIE Conf. Ser., 6272

Caswell, J. L., Vaile, R. A., Ellingsen, S. P., Whiteoak, J. B., \& Norris, R. P. 1995, MNRAS, 272, 96

Cesaroni, R., Walmsley, C. M., \& Churchwell, E. 1992, A\&A, 256, 618

Chini, R., Hoffmeister, V. H., Nasseri, A., Stahl, O., \& Zinnecker, H. 2012, MNRAS, 424, 1925

Connelley, M. S., Reipurth, B., \& Tokunaga, A. T. 2008, AJ, 135, 2496

Cyganowski, C. J., Whitney, B. A., Holden, E., et al. 2008, AJ, 136, 2391

De Buizer, J. M., Radomski, J. T., Telesco, C. M., \& Piña, R. K. 2005, ApJS, 156,179

Herriot, G., Morris, S., Anthony, A., et al. 2000, in SPIE Conf. Ser. 4007, ed. P. L. Wizinowich, 115

Hester, J. J., \& Desch, S. J. 2005, in Chondrites and the Protoplanetary Disk, ASP Conf. Ser., eds. A. N. Krot, E. R. D. Scott, \& B. Reipurth, 341, 107

Hodapp, K. W., Jensen, J. B., Irwin, E. M., et al. 2003, PASP, 115, 1388

Hunter, T. R., Phillips, T. G., \& Menten, K. M. 1997, ApJ, 478, 283

Konigl, A., \& Pudritz, R. E. 2000, Protostars and Planets IV, 759

Kraus, S., Balega, Y., Elitzur, M., et al. 2006, A\&A, 455, 521

Kuchar, T. A., \& Bania, T. M. 1994, ApJ, 436, 117

Linz, H., Stecklum, B., Henning, T., Hofner, P., \& Brandl, B. 2005, A\&A, 429, 903

Mundt, R., Buehrke, T., Solf, J., Ray, T. P., \& Raga, A. C. 1990, A\&A, 232, 37

Ortega, M. E., Paron, S., Cichowolski, S., Rubio, M., \& Dubner, G. 2012, A\&A, 546, A96

Papaloizou, J. C. B., \& Terquem, C. 1995, MNRAS, 274, 987

Paron, S., Cichowolski, S., \& Ortega, M. E. 2009, A\&A, 506, 789

Peters, T., Banerjee, R., Klessen, R. S., et al. 2010, ApJ, 711, 1017

Preibisch, T., Balega, Y. Y., Schertl, D., \& Weigelt, G. 2003, A\&A, 412, 735

Qin, S.-L., Wang, J.-J., Zhao, G., Miller, M., \& Zhao, J.-H. 2008, A\&A, 484, 361

Reipurth, B., \& Bally, J. 2001, ARA\&A, 39, 403

Reipurth, B., Yu, K. C., Heathcote, S., Bally, J., \& Rodríguez, L. F. 2000, AJ, 120,1449

Shu, F. H., Najita, J., Ostriker, E. C., \& Shang, H. 1995, ApJ, 455, L155

Smith, M. D., \& Rosen, A. 2005, MNRAS, 357, 579

Stahler, S. W., \& Palla, F. 2005, The Formation of Stars (Wiley-VCH)

Urquhart, J. S., Hoare, M. G., Purcell, C. R., et al. 2009, A\&A, 501, 539

Vaidya, B., Fendt, C., Beuther, H., \& Porth, O. 2011, ApJ, 742, 56

Weigelt, G., Beuther, H., Hofmann, K.-H., et al. 2006, A\&A, 447, 655

Wilner, D. J., Ho, P. T. P., \& Zhang, Q. 1996, ApJ, 462, 339

Wood, D. O. S., \& Churchwell, E. 1989, ApJS, 69, 831

Wu, Y., Wei, Y., Zhao, M., et al. 2004, A\&A, 426, 503 